\title{
Arm weight support training improves functional motor outcome and movement smoothness after stroke
}

\author{
Michelangelo Bartolo, MD, PhDa,b \\ Alessandro Marco De Nunzio, PhD ${ }^{a, b}$ \\ Fabio Sebastiano, PhD $^{c}$ \\ Francesca Spicciato, $\mathbf{P T}^{\mathrm{a}}$ \\ Paolo Tortola, PTa \\ Jan Nilsson, $\mathrm{PhD}^{\mathrm{d}}$ \\ Francesco Pierelli, MDa,e
}

\author{
a Neurorehabilitation Unit, IRCCS NEUROMED, \\ Pozzilli (Isernia), Italy \\ b "INM-MotionLab" Laboratory of Multifactorial \\ Biomechanics - Neurorehabilitation Unit, IRCCS \\ NEUROMED, Pozzilli (Isernia), Italy \\ c Bioengineering Unit, IRCCS NEUROMED, Pozzilli \\ (Isernia), Italy \\ ${ }^{d}$ Laboratory of Biomedical Engineering, Salvatore \\ Maugeri Foundation IRCCS, Scientific Institute of \\ Castel Goffredo (MN), Italy \\ - Department of Medico-Surgical Sciences and \\ Biotechnologies, Sapienza University of Rome, \\ Italy
}

Correspondence to: Michelangelo Bartolo E-mail: bartolomichelangelo@gmail.com

\section{Summary}

The aim of this study was to compare the effectiveness in acute stroke patients of a rehabilitation program performed with or without an arm weight support device. Twenty-eight acute, first-ever unilateral stroke patients were enrolled in a single-blind, randomized controlled trial.

Clinical evaluation included Fugl-Mayer Assessment, Functional Independence Measure and kinematic analysis [maximum and mean hand velocity, maximum range of motion (Max RoM), normalized jerk (NJ)]. Patients received 12 daily 30-minute sessions (6/week) of additional upper limb therapy performed using an arm weight support device (study group) or additional traditional physiotherapy (control group). The patients were evaluated on admission and at the end of the rehabilitation intervention.

The two groups were clinically comparable on admission ( $p>0.05$ ). Both groups showed significant improvements in clinical scale scores and in Max RoM in flexion-extension, while only the study group showed improvements in NJ and in Max RoM in adductionabduction.
Rehabilitation training using an arm weight support device appears to be a useful method to supplement conventional therapy in acute stroke patients, increasing smoothness of movement and motor function.

KEY WORDS: arm weight support, kinematics, neurorehabilitation, stroke, upper limb

\section{Introduction}

Hemiparesis has been reported to be the principal sequela of stroke, occurring in more than $80 \%$ of all cases (Sommerfeld et al., 2004). Studies indicate that only 5 to $20 \%$ of stroke patients with hemiparesis regain upper extremity function (Kwakkel et al., 2003; Kollen et al., 2006) and that only $6 \%$ are satisfied with the level of functionality of the affected upper limb (Broeks et al., 1999).

Although the degree of motor function recovery after stroke is strongly related to the site and severity of the lesion, the recovery process may be stimulated and molded by rehabilitation programs using various techniques and exercises designed to promote motor relearning (Stein et al., 2007; Oujamaa et al., 2009). However, because of the limited success of traditional rehabilitation programs in restoring upper extremity function after stroke, researchers have been searching for other solutions, including ones integrating advanced technologies and electromechanical devices (Housman et al., 2009; Huang and Krakauer, 2009; Backus et al., 2010; Mehrholz et al., 2012; Zariffa et al. 2012).

These devices allow time-specific tasks to be executed repeatedly in a controlled and reliable way, as this has been shown to be a determining factor in facilitating cortical reorganization, allowing a concomitant increase in motor ability and improved functional activity performance (Liepert, 2006).

Recent evidence has shown that intensive repetition of movements could make a useful contribution to clinical practice (Kawahira et al., 2010), constituting a promising approach for patients affected by motor impairments due to neurological diseases, such as multiple sclerosis (Gijbels et al., 2011), cervical spinal cord injuries (Zariffa et al., 2012), and chronic stroke (Krebs et al., 2002; Macclellan et al., 2005; Sanchez et al., 2006; Masiero et al., 2007; Colombo et al., 2008; Kwakkel et al., 2008). Indeed, the use of devices designed to intensify therapy in the single patient could be a promising field of investigation (Masiero et al., 2009). From this perspective, it is well known that the acute phases after neurological injury are the most sensitive to approaches designed to 
exploit neural plasticity (Paolucci et al., 2000; Dobkin, 2004).

Even though previous studies, exploring the servoactuated robotic rehabilitation approach, confirmed an improvement of proximal arm functions (Krebs et al., 2002), optimal extension of this motor recovery to the distal motor segments of the upper limb remains to be demonstrated (Wisneski and Johnson, 2007). Conversely, arm weight support rehabilitation based on the use of passive orthotic devices has been shown to improve features of hand movements that may be considered crucially important for achieving a positive recovery of upper limb motor control (Morasso, 1981; Wisneski and Johnson, 2007) and may be related to enhanced functional ability.

Moreover, while robotic actuators may decrease patients' levels of effort and attention, having negative effects on neural plasticity, passive arm orthoses allow them to perform semiautonomous rehabilitation training programs (Housman et al., 2009).

The aim of this study was to evaluate, in a randomized controlled trial design, the effectiveness in acute stroke inpatients of a program of motor rehabilitation, designed to promote recovery of upper limb function.

\section{Materials and methods}

\section{Patients}

The study included 38 inpatients (15 F, 23 M) consecutively referred to the Neurorehabilitation Unit at our institution.

The inclusion criteria were: i) a diagnosis of first-ever stroke (ischemic or hemorrhagic), confirmed by neuroimaging (CT or $\mathrm{MRI}$ ); ii) age between 21 and 85 years; iii) an interval of no more than four weeks since the acute event; iv) trunk control corresponding to a Sitting Balance score $\geq 2$ (sitting position maintained without difficulty, but assistance needed in all the strengthening tasks); v) upper limb paresis $\geq 2 / 5$ Medical Research Council (MRC) evaluated in wristhand and shoulder-upper arm; vi) no serious cognitive deficits [Mini Mental State Examination (MMSE) $\geq 25$ (Folstein et al., 1975)].

Exclusion criteria were: i) clinical or functional contraindication to intensive treatment (e.g.: muscle contractures, dystonia); ii) upper limb pain [Visual Analog Scale (VAS) score $\geq 4 / 10$ ]; iii) spasticity, [Modified Ashworth Scale (MAS) score $\geq 2$ ]; iv) decubitus or pressure sores on the wrist or hand; v) visual neglect. All the participants gave their written informed consent to participate in the study.

The study was conducted in accordance with the revised version of Declaration of Helsinki and all procedures in the protocol were fully approved by the local ethics committee.

\section{Rehabilitation program}

The rehabilitation training was additional to a six days/week intensive rehabilitation program, based on 60 minutes/day of individual sessions of conventional physiotherapy (Bobath, Kabat, etc.).

All patients received 12 daily 30 -minute sessions (6/week) of additional upper limb training therapy performed using an arm weight support device (study group, SG) or additional conventional physiotherapy including passive mobilization exercises, active motor exercises specifically designed to enhance proprioceptive and sensory stimulation, and exercises to improve fine motor function of the hand (control group, CG).

The SG used an instrumented arm orthosis with a spring mechanism allowing adjustable arm weight support (ArmeoßSpring, Hocoma AG, Switzerland). This device can generate assistive forces of up to $66 \mathrm{~N}$ in flexion/extension and up to $30 \mathrm{~N}$ for the arm and forearm respectively. These forces are far from the maximum forces that a healthy subject can generate, but the functional support they provide is sufficient to allow patients to perform challenging exercises that simulate typical activities of daily living (ADL).

The arm orthosis integrates seven angle sensors and one pressure sensor (hand gripper sensor) that allow direct interaction between the motion of the patient's arm and the associated therapy software (Armeo ${ }^{\circledR}$ Control), which monitors sensor movement in real time. The software is based on a series of stimulating games and exercises designed to engage patients in volitional (e.g. cleaning a window or stove) or goal-directed tasks (catching falling water in a mug or pointing at unpredictable visual stimuli, i.e. ladybugs popping up on the screen).

\section{Outcome measures}

Clinical and functional scales were used to evaluate patients' motor performance before (T0) and after (T1) the program of 12 daily sessions.

All assessments were performed by a neurologist and a physiotherapist not involved in the rehabilitation program and blinded to the groups to which the patients were assigned.

The Fugl-Meyer system (FM) was used for motor assessment of the upper extremity (FM score range: 0-66) (Fugl-Meyer et al., 1975), while the Functional Independence Measure (FIM, range 18-126) (Kidd et al., 1995) was used for global functional evaluation. To better disclose the "ecological" (real-life) impact of upper limb motor recovery, the FIM self-care score was used. This score was defined as the sum of the following items: eating, grooming, bathing, dressingupper body, dressing-lower body, toileting.

In order to evaluate spasticity changes, the MAS (range 0-5) was administered, considering the shoulder abductors and the elbow, wrist, finger and thumb flexors.

In addition, pain was assessed using a VAS score (range $0-10$ ). At the end of treatment, the subjects rated their level of satisfaction with the therapy (1=poor; $2=$ sufficient; $3=$ good; 4=excellent) (Lambercy et al., 2011). 


\section{Kinematic evaluation}

Before and after the rehabilitation training, all the enrolled subjects underwent a kinematic evaluation of the affected arm. This was performed using the Armeo ${ }^{\circledR C}$ Control software and an infrared camerabased optoelectronic system (BTS Smart DX5000 BTS Bioengineering, Milan, Italy).

All patients received $40 \%$ arm weight support during the rehabilitation training and/or the evaluation sessions.

To track the movements of the patient's affected arm, a set of three infrared reflective markers was placed on the two acromial bones (shoulder markers) and on the Armeo system manipulator (hand marker) (Fig. 1). To perform the instrumental evaluation the Armeo®Spring system was set up in a movement analysis laboratory.

Before the evaluation session, each subject was instructed in the correct way to execute two different motor tasks and then trained for about 10 minutes. These tasks were: i) arm flexion-extension and abduction-adduction to reach the maximum range of motion; ii) a reaching motor task consisting of pointing at unpredictable popping-up visual stimuli, starting from an anatomically centered position, reported as the origin of the Cartesian $X$ and $Y$ axes, which were oriented, respectively, parallel with and vertically to the ground (Fig. 1).

During the motor tasks, 3D hand marker movements were acquired. Subsequently, the hand marker movements were projected in the $X-Y$ plane and analyzed off-line.

The following indices were calculated to evaluate upper limb motor performance:

- 2D maximum range of motion (Max RoM) (cm): shoulder abduction-adduction and flexion-extension Max RoM along the horizontal $(\mathrm{X})$ and vertical $(\mathrm{Y})$ axes, respectively.

- maximum and mean velocity (Max Vel, Mean Vel) $(\mathrm{cm} / \mathrm{s})$ : maximum and mean values of the ratio between the path length $(L)$ and time duration $(T)$ of every catching/returning movement. $\mathrm{L}$ and $\mathrm{T}$ values were calculated considering a $5 \%$ velocity threshold in reaching the popping-up visual stimuli and returning to the centered position. The velocity threshold was $5 \%$ of the maximum velocity achieved by the subject during the whole second motor task session.

- normalized jerk (NJ) (without dimension): square root of the jerk $[j(t)$, norm of the third time derivative of the hand marker position], averaged over the entire catching/returning movement duration and normalized with respect to $T$ and $L$ (Casadio et al., 2010). This measure is sensitive to smoothness, with larger jerk values corresponding to less smoothness.

Normalized jerk $=\sqrt{ }\left[1 / 2 \times \frac{\left(\int j(t)^{2} d t\right) \times T^{5}}{L^{2}}\right]$ (Teulings et al., 1997).

\section{Statistical analysis}

The means of the kinematic indices and clinical scores were calculated for each patient considering the motor

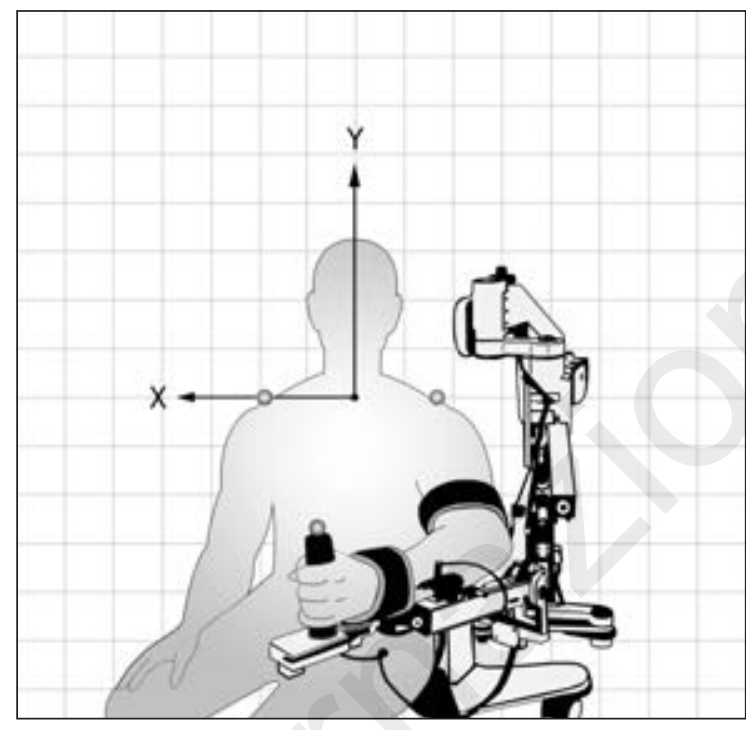

Figure 1 - Arm weight support device and marker positioning. Gray spheres: two shoulder markers and a hand marker. The Cartesian reference system ( $X$ and $Y$ axes) is embedded in a vertical-to-the ground plane in which hand marker movements were projected. The midpoint of the shoulder markers corresponds to the origin of the reference axes.

tasks performed during the two evaluation sessions. A between-groups analysis to compare demographic and clinical characteristics and kinematic indices at admission and at the end of the rehabilitation program was performed using the unpaired Student's t-test. The paired Student's t-test was used to compare the evaluation sessions (T0 vs T1) in both the CG and the SG. To provide evidence of the relative increase in arm motor function for both groups, the gain for kinematic indices was calculated as the T1-T0 difference and compared between groups using the unpaired Student's t-test.

Statistical analysis was performed using STATISTICA 7.1 (StatSoft $®$, Italia Srl, Vigonza, PD, Italy). The threshold for significance was set at $p<0.05$.

\section{Results}

\section{Clinical measures}

Of the 38 patients enrolled in the study, $28(10 \mathrm{~F}, 18 \mathrm{M}$; mean age: $57.3 \pm 11.2$ years) met the inclusion criteria and were assigned to the CG or SG by means of a software-based randomization process (Fig. 2, over). Baseline demographic, clinical characteristics and kinematic indices did not reveal any statistically significant differences between the two groups at admission (T0). The FM score did not show a statistically significant change (T1 vs T0) in either group.

Analysis of the FIM total score and FIM self-care score revealed significant improvements (T1 vs T0) in both groups (Table I, over). 


\section{Bartolo et al.}

No statistical significance was found on comparing the outcome measures of the two groups at T1.

No pain was reported on admission in either group, and no changes were found at $\mathrm{T} 1$.

Two patients in the SG presented spasticity on admission: the first one had a MAS score of 1 at the elbow and 1+ at the wrist; while the second had a MAS score of 1 both at the shoulder and at the elbow. No changes were found in the neurophysiological scores after the treatment.

The satisfaction ratings showed a good level of satisfaction with the therapy in the SG $(3.16 \pm 0.75)$ and sufficient satisfaction in the CG $(2.57 \pm 0.53)$.

\section{Kinematic indices}

All the kinematic indices at T0 and T1 were compared for both CG and SG (Fig. 3).
While the Max Vel and Mean Vel of reaching movements showed no statistically significant change in either the CG or the SG, the Max RoM during flexionextension movements was found to be significantly improved in both groups.

Patients in the SG also showed a statistically significant improvement in max RoM in the horizontal plane (abduction-adduction movements) and a significant reduction in NJ.

The gain for max RoM in abduction-adduction and flexion-extension, and for NJ was significantly greater in the SG than in the CG (Fig. 4).

\section{Discussion}

This study examined the effectiveness of rehabilitation training performed with or without an arm weight sup-

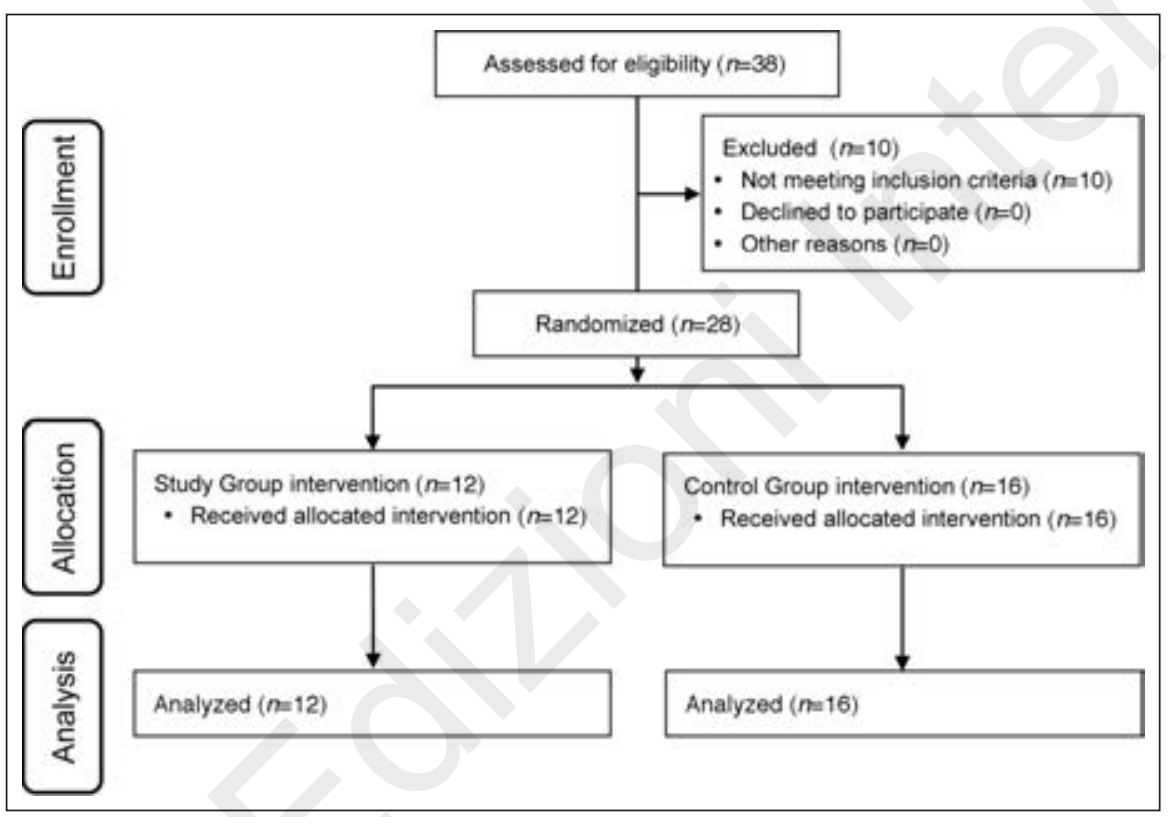

Figure 2 - CONSORT flow diagram.

Table I - Demographic and clinical measures in patients undergoing rehabilitation training with or without an arm support device.

\begin{tabular}{llclc}
\hline & Control Group & T1 vs T0 $(p)$ & Study Group & T1 vs T0 $(p)$ \\
\hline Age, years & $51.2 \pm 7.0$ & - & $63.5 \pm 11.6$ & - \\
Sex (males/females) & $10 / 6$ & - & $8 / 4$ & - \\
Lesion side (right/left) & $9 / 7$ & - & $6 / 6$ & - \\
Stroke type (hemorrhagic/ischemic) & $2 / 14$ & - & $2 / 10$ & - \\
FIM total T0 & $43.1 \pm 19.6$ & & $40.3 \pm 10.0$ & 0.025 \\
FIM total T1 & $83.6 \pm 30.5$ & 0.001 & $77.1 \pm 27.2$ & \\
FIM self-care T0 & $7.0 \pm 3.3$ & 0.012 & $19.1 \pm 2.0$ & 0.035 \\
FIM self-care T1 & $19.8 \pm 9.9$ & & $22.6 \pm 21.8$ & n.s. \\
FM T0 & $23.5 \pm 15.6$ & n.s. & $29.3 \pm 21.7$ & \\
FM T1 & $27.8 \pm 18.9$ & &
\end{tabular}

FIM=Functional Independence Measure; n.s.=not significant. Values are mean \pm SD or number. 
port device in a homogenous cohort of acute stroke inpatients. At the end of treatment, the clinical scores (FIM total, FIM self-care) were improved in both the $S G$ and the CG.

The kinematic analysis revealed a statistically significant enhancement of the Max RoM along vertical axes in both groups, while improvements in the Max RoM along horizontal axes and in $\mathrm{NJ}$ were observed only in the SG.

Although previous studies conducted in chronic stroke patients have reported an increased functional motor gain after arm-weight support device training (Krebs et al., 2002; Macclellan et al., 2005; Sanchez et al., 2006; Masiero et al., 2007; Colombo et al., 2008; Kwakkel et al., 2008), the present study was the first to be conducted during the acute phase after stroke, using kinematic analysis to evaluate upper limb motor performance. It is strongly suggested in the literature that the acute phases following neurological damage are the most sensitive to approaches designed to exploit neural plasticity (Paolucci et al., 2000; Dobkin, 2004), particularly the first six months after stroke (Paolucci et al., 2000). Moreover, it has been suggested that stroke patients obtain a better functional outcome when rehabilitation training was initiated immediately after the stroke, and the training was based on intensive multisensory stimulation (Masiero et al., 2009). In fact, the use of the arm weight support device could be a means of enriching the sensorimotor stream, allowing active repetitive training with arm motion biofeedback in a patient-virtual environment interaction. These observations were confirmed in recent studies, in which robotic/electromechanical devices enabled practice of independent and repetitive movements, particularly in a motivational context enriched by virtual reality, as in this study (Backus et al., 2010, Mehrholz et al., 2012).

Movement smoothness has been used as a measure of motor performance in both healthy subjects and patients affected by stroke (Platz et al., 1994; Kahn et
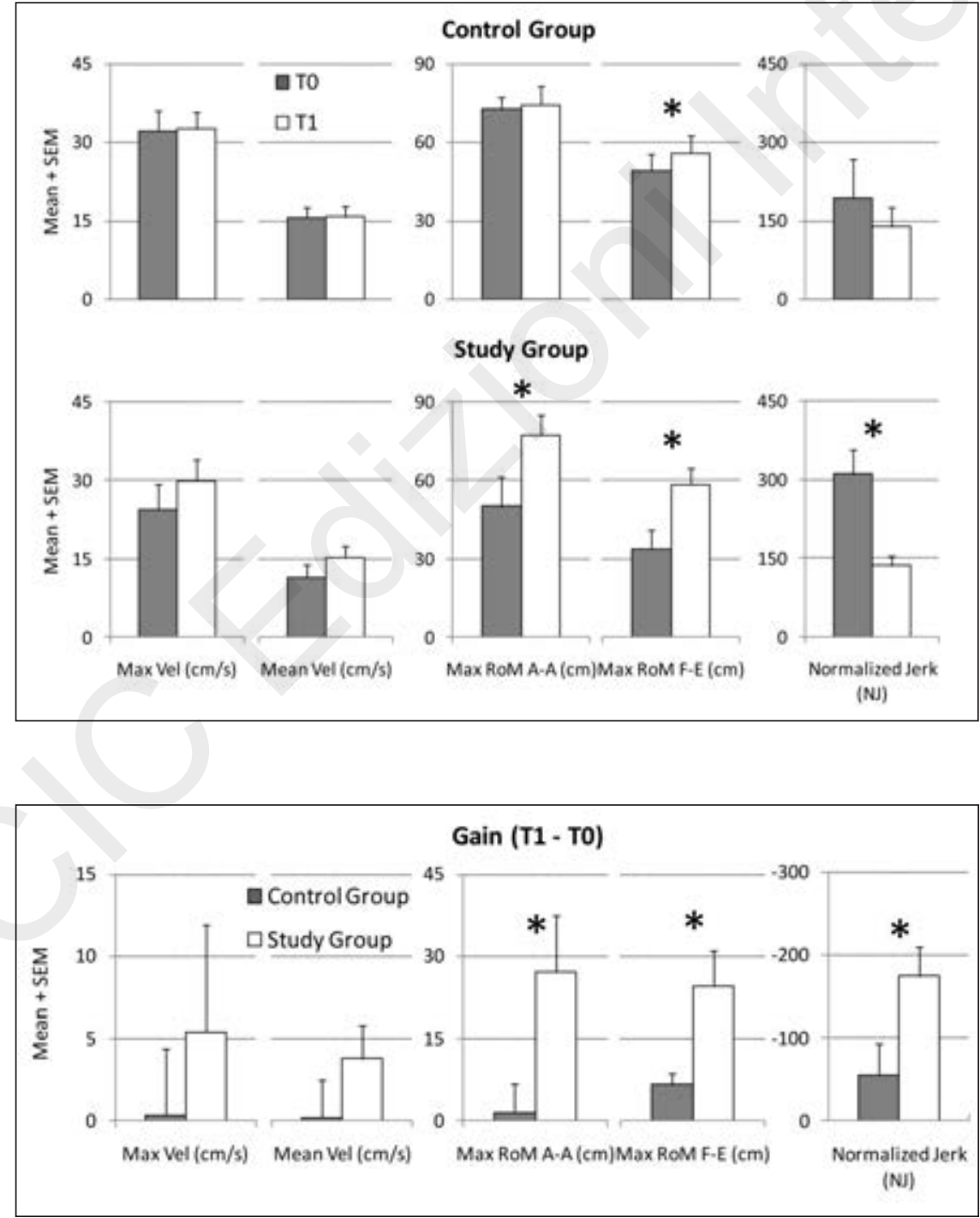

Figure 3 - Mean + standard error mean (error bars) of the kinematic indices for upper limb motor performance evaluation.

Abbreviations: Max Vel= maximum velocity; Mean Vel=mean velocity; Max RoM $A A=$ maximum range of motion in abduction-adduction; Max RoM FE=maximum range of motion in flexion-extension. Gray columns: before rehabilitation treatment (TO); white columns: after rehabilitation treatment (T1). The asterisks represent significant differences.
Figure 4 - Mean + standard error mean (error bars) of differences in kinematics indices used for upper-limb motor performance evaluation between the evaluation sessions after and before rehabilitation treatment ( $\mathrm{T} 1$ and T0, respectively).

Abbreviations: Max Vel= maximum velocity; Mean Vel=mean velocity; Max RoM $A A=$ maximum range of motion in abduction-adduction; Max RoM FE=maximum range of motion in flexion-extension. The asterisks represent significant differences. 
al., 2006). Smoothness is a critical characteristic of skilled, coordinated human movements, and it is a distinct independent measure with respect to speed and distance.

Studies of development and recovery from neural injury strongly suggest that smoothness is a result of learned coordinative processes rather than a natural consequence of the structure of the neuromuscular system (Levin, 1996).

Jerk, i.e. the time derivative of acceleration, has been used as an empirical measure of movement smoothness (Casadio et al., 2010).

In this study, NJ was tested as a measure of the quality of selective motor control and was used to evaluate the qualitative features of the multi-joint upper limb movements, considering its inverse correlation with the smoothness of hand movement (Flash and Hogan, 1985; Caimmi et al., 2008). Our data bring out additional evidence regarding the recovery process after neurological damage, which is reflected in the execution of progressively smoother movements as confirmed by a significant reduction in NJ (Krebs et al., 1998).

Moreover, while robotic actuators may decrease patients' effort and attention, having, in turn, negative effects on neural plasticity, passive arm orthoses and arm-weight support devices both make it possible to perform semiautonomous rehabilitation training, inducing more natural active arm control and increasing the smoothness of reaching tasks.

When moving the hand between pairs of targets, patients tend to generate roughly straight hand trajectories with a single-peaked, bell-shaped speed profile (Morasso, 1981); this behavior was independent of the part of the work-space in which the movement was performed and these results are strong indications that planning takes place in terms of hand trajectories rather than joint rotations (Flash and Hogan, 1985).

Therefore, the kinematic indices analyzed provided a quantitative indication of upper limb motor function control.

Whereas the velocity indices did not change over the rehabilitation period, independently of the rehabilitation approach, a significant improvement in hand range of motion was achieved by the patients in the SG. These results indicate a functional improvement obtained with arm weight support therapy, and therefore the possibility of achieving a wider set of movements directly related to a wider work-space dimension. Considering that the second motor task did not make provision for an auditory cue for the catching/ returning movements, the patients could not be driven to execute faster movements than they would normally have executed, and therefore, velocity indices could not be used to reveal functional motor outcome improvements.

Previous studies concerning robotic rehabilitation approaches in stroke patients have reported improved proximal arm functions (Burgar et al., 2000; Krebs et al. 2002), but did not show an optimal motor recovery of the distal motor segments of the upper limb, which are involved in most ADL (Wisneski and Johnson, 2007). Conversely, arm weight support rehabilitation leads to reduced $\mathrm{NJ}$ and to increased hand RoM, which is crucially important for positive recovery of upper limb motor control (Morasso, 1981; Wisneski and Johnson, 2007), and possibly related to improved functional ability in executing ADL.

The therapy satisfaction score revealed a good level of satisfaction among the patients in the SG $(3.16 \pm 0.75)$ and sufficient satisfaction in the CG $(2.57 \pm 0.53)$. These positive data could be due to the unweighting effect of the robotic exoskeleton, and also to the advantages of conducting exercises in a motivating and stimulating virtual reality setting (Backus et al., 2010; Saposnik and Levin, 2011; Mehrholz et al., 2012). The arm weight support device (Armeo®Spring) in fact, incorporates a series of volitional and functional exercises that engage the patient in sessions of meaningful games based on daily life activities, which can be programmed with a custom-calibrated range of motion to a suitable level of difficulty. It is also possible that patients, in this virtual reality game context, are motivated to improve their "learning curve".

There are two main limitations of this study. The first of these is the small sample size; however, the data may be regarded as preliminary; a subsequent study with a larger population will be performed to confirm and extend the present results.

The second limitation is the lack of follow-up data for establishing the extent to which the motor recovery is maintained in daily activities after returning home. This study focused on the early phase after stroke, and the self-care activities (FIM self-care score) were considered as a measure of the functional impact of the training. As of now, longitudinal assessments are being collected to test both the maintenance of the learned motor skills, and their transfer into "ecologic" (real-life) contexts.

In conclusion, these results show the effectiveness of arm weight support systems in upper limb recovery in the acute stages after stroke, providing further experimental evidence that individuals receiving therapy with this kind of device can improve their movement ability. Technological and robotic devices are opening up new opportunities and therapies that might enhance conventional approaches, however the definition of clinical indications for proper use of these new devices will be crucial to ensure more effective treatments.

\section{Acknowledgments}

This study was supported by " $5 \mathrm{x}$ mille" donations.

\section{References}

Backus D, Winchester P, Tefertiller C (2010). Translating research into clinical practice: integrating robotics into neurorehabilitation for stroke survivors. Top Stroke Rehabil 17: 362-370.

Broeks JG, Lankhorst GJ, Rumping K et al (1999). The longterm outcome of arm function after stroke: results of a follow-up study. Disabil Rehabil 21: 357-364.

Burgar CG, Lum PS, Shor PC et al (2000). Development of 
robots for rehabilitation therapy: the Palo Alto VA/Stanford experience. J Rehabil Res Dev 37: 663-673.

Caimmi M, Carda S, Giovanzana C et al (2008). Using kinematic analysis to evaluate constraint-induced movement therapy in chronic stroke patients. Neurorehabil Neural Repair 22: 31-39.

Casadio M, Pressman A, Fishbach A et al (2010). Functional reorganization of upper-body movement after spinal cord injury. Exp Brain Res 207:233-247.

Colombo R, Pisano F, Micera S et al (2008). Assessing mechanisms of recovery during robot-aided neurorehabilitation of the upper limb. Neurorehabil Neural Repair 22: 50-63.

Dobkin BH (2004). Strategies for stroke rehabilitation. Lancet Neurol 3: 528-536.

Flash T, Hogan N (1985). The coordination of arm movements: an experimentally confirmed mathematical model. J Neurosci 5: 1688-1703.

Folstein MF, Folstein SE, McHugh PR (1975). "Mini-mental state". A practical method for grading the cognitive state of patients for the clinician. J Psychiatr Res 12: 189-198.

Fugl-Meyer AR, Jääskö L, Leyman I et al (1975). The poststroke hemiplegic patient. 1. a method for evaluation of physical performance. Scand J Rehabil Med 7: 13-31.

Gijbels D, Lamers I, Kerkhofs L et al (2011). The Armeo Spring as training tool to improve upper limb functionality in multiple sclerosis: a pilot study. J Neuroeng Rehabil 8: 5.

Housman SJ, Scott KM, Reinkensmeyer DJ (2009). A randomized controlled trial of gravity-supported, computerenhanced arm exercise for individuals with severe hemiparesis. Neurorehabil Neural Repair 23: 505-514.

Huang VS, Krakauer JW (2009). Robotic neurorehabilitation: a computational motor learning perspective. J Neuroeng Rehabil 6: 5.

Kahn LE, Zygman ML, Rymer WZ, et al (2006). Robot-assisted reaching exercise promotes arm movement recovery in chronic hemiparetic stroke: a randomized controlled pilot study. J Neuroeng Rehabil 3: 12.

Kawahira K, Shimodozono M, Etoh S, et al (2010). Effects of intensive repetition of a new facilitation technique on motor functional recovery of the hemiplegic upper limb and hand. Brain Inj 24:1202-1213.

Kidd D, Stewart G, Baldry J et al (1995). The Functional Independence Measure: a comparative validity and reliability study. Disabil Rehabil 17: 10-14.

Kollen B, Kwakkel G, Lindeman E (2006). Functional recovery after stroke: a review of current developments in stroke rehabilitation research. Rev Recent Clin Trials 1: 75-80.

Krebs HI, Hogan N, Aisen ML, et al (1998). Robot-aided neurorehabilitation. IEEE Trans Rehabil Eng 6: 75-87.

Krebs HI, Volpe BT, Ferraro M et al (2002). Robot-aided neurorehabilitation: from evidence-based to science-based rehabilitation. Top Stroke Rehabil 8: 54-70.

Kwakkel G, Kollen BJ, Krebs HI (2008) Effects of robot-assisted therapy on upper limb recovery after stroke: a systematic review. Neurorehabil Neural Repair 22: 111-121.

Kwakkel G, Kollen BJ, van der Grond J et al (2003). Probability of regaining dexterity in the flaccid upper limb: impact of severity of paresis and time since onset in acute stroke. Stroke 34: 2181-2186.

Lambercy O, Dovat L, Yun H et al (2011). Effects of a robot- assisted training of grasp and pronation/supination in chronic stroke: a pilot study. J Neuroeng Rehabil 8: 63.

Levin MF (1996). Interjoint coordination during pointing movements is disrupted in spastic hemiparesis. Brain 119 ( Pt 1): $281-293$.

Liepert J (2006). Motor cortex excitability in stroke before and after constraint-induced movement therapy. Cogn Behav Neurol 19:41-47.

Macclellan LR, Bradham DD, Whitall J et al (2005). Robotic upper-limb neurorehabilitation in chronic stroke patients. $J$ Rehabil Res Dev 42: 717-722.

Masiero S, Celia A, Rosati G et al (2007). Robotic-assisted rehabilitation of the upper limb after acute stroke. Arch Phys Med Rehabil 88:142-149.

Masiero S, Rosati G, Valarini S et al (2009). Post-stroke robotic training of the upper limb in the early rehabilitation phase. Funct Neurol 24:203-206.

Mehrholz J, Hädrich A, Platz T et al (2012). Electromechanical and robot-assisted arm training for improving generic activities of daily living, arm function, and arm muscle strength after stroke. Cochrane Database Syst Rev 6: CD006876.

Morasso P (1981). Spatial control of arm movements. Exp Brain Res 42:223-227.

Oujamaa L, Relave I, Froger J et al (2009). Rehabilitation of arm function after stroke. Literature review. Ann Phys Rehabil Med 52: 269-293.

Paolucci S, Antonucci G, Grasso MG et al (2000). Early versus delayed inpatient stroke rehabilitation: a matched comparison conducted in Italy. Arch Phys Med Rehabil 81: 695700.

Platz T, Denzler P, Kaden B et al (1994). Motor learning after recovery from hemiparesis. Neuropsychologia 32: 12091223.

Sanchez RJ, Liu J, Rao S et al (2006). Automating arm movement training following severe stroke: functional exercises with quantitative feedback in a gravity-reduced environment. IEEE Trans Neural Syst Rehabil Eng 14: 378389.

Saposnik G, Levin M; Outcome Research Canada (SORCan) Working Group (2011). Virtual reality in stroke rehabilitation: a meta-analysis and implications for clinicians. Stroke 42: $1380-1386$.

Sommerfeld DK, Eek EU, Svensson AK et al (2004). Spasticity after stroke: its occurrence and association with motor impairments and activity limitations. Stroke 35: 134-139.

Stein J, Narendran K, McBean J et al (2007). Electromyographycontrolled exoskeletal upper-limb-powered orthosis for exercise training after stroke. Am J Phys Med Rehabil 86: 255-261.

Teulings HL, Contreras-Vidal JL, Stelmach GE et al (1997). Parkinsonism reduces coordination of fingers, wrist, and arm in fine motor control. Exp Neurol 146: 159-70.

Wisneski KJ, Johnson MJ (2007). Quantifying kinematics of purposeful movements to real, imagined, or absent functional objects: implications for modelling trajectories for robot-assisted ADL tasks. J Neuroeng Rehabil 4: 7.

Zariffa J, Kapadia N, Kramer JL et al (2012). Feasibility and efficacy of upper limb robotic rehabilitation in a subacute cervical spinal cord injury population. Spinal Cord 50:220-226. 